\title{
Prioritization of Noise Control Methods by the Analytical Hierarchy Process (AHP) in a Battery Factory
}

\author{
Ayub Abdullahzadeh Thani ${ }^{1}$, Rasoul Yarahmadi $^{2}$, Jamileh Abolghasemi ${ }^{3}$, Mehran \\ Firoozbakhsh $^{4}$, Jafar Besharati ${ }^{5}$, Iraj Alimohammadi6,* \\ ${ }^{1}$ MSc, Department of Occupational Health Engineering, Faculty of Health, Iran University of Medical Sciences, Tehran, Iran \\ 2 Professors Department of Occupational Health Engineering, School of Health, Iran University of Medical Sciences, Tehran, Iran \\ ${ }^{3}$ Associate Professor, Department of Biostatistics, School of Health, Iran University of Medical Sciences, Tehran, Iran \\ ${ }^{4}$ MSc, Department of Environmental Engineering, Tehran Science and Research Branch, Islamic Azad University, Tehran, Iran \\ ${ }^{5}$ MSc, Department of Environmental Management, Tehran Science and Research Branch, Islamic Azad University, Tehran, Iran \\ ${ }^{6}$ Professors, Department of Occupational Health Engineering, School of Health, Iran University of Medical Sciences, Tehran, Iran \\ * Corresponding Author: Iraj Alimohammadi, Department of Occupational Health Engineering, School of Health, Iran University \\ of Medical Sciences, Tehran, Iran. Email: irajrastin1@gmail.com
}

Received: $26 / 01 / 2021$ Accepted: 07/03/2021

How to Cite this Article: Abdullahzadeh Thani A, Yarahmadi R, Abolghasemi J, Firoozbakhsh M, Besharati J, Alimohammadi I. Prioritization of Noise Control Methods by the Analytical Hierarchy Process (AHP) in a Battery Factory. J Occup Hyg Eng. 2021; 8(1): 65-73. DOI: 10.29252/johe 8.1 .65

\section{Abstract}

Background and Objective: Due to major constraints on time, implementation, and funding in every industry, one of the essential strategies for the management of noise control and selection of the best method is to prioritize noise control methods. The present study aimed to prioritize noise control methods in a battery factory using the Analytical Hierarchy Process (AHP).

Materials and Methods: The present study was conducted based on a descriptive-analytical cross-sectional design. After measurement of sound pressure level and identification of the main sources of noise pollution, Content Validity Index (CVI) and Content Validity Ratio (CVR) were used for screening the criteria and methods of noise control. The relative weight of each of them was calculated by two factors of Eigenvalue and Eigenvector to prioritize noise control methods based on the study criteria.

Results: Based on the results, the Inconsistency Ratio (IR) in pairwise comparisons in all cases was less than $10 \%$, and the consistency of the answers was confirmed. Moreover, among the study criteria, the acoustic efficiency of method with weight $(0.1810)$, and among the proposed methods for noise control, the method of controlling the time of exposure to noise and training of workers with weight (0.1732) had the highest priority.

Conclusion: As evidenced by the obtained results, the best criterion for selecting a sound control device is the acoustic efficiency of the method. Furthermore, based on the results, controlling the duration of exposure to noise and training workers in conditions of high noise exposure was the best method to control noise in a battery factory.

Keywords: Analytical Hierarchy Process; Battery Factory; Noise Control; Noise Pollution; Prioritizing 
dof: $10.29252 /$ johe.8.1.65

\section{اولويتبندى روشهاى كنترل صدا با استفاده از فرايند تحليل سلسله مراتبى (AHP) در يك صنعت باترىسازى}

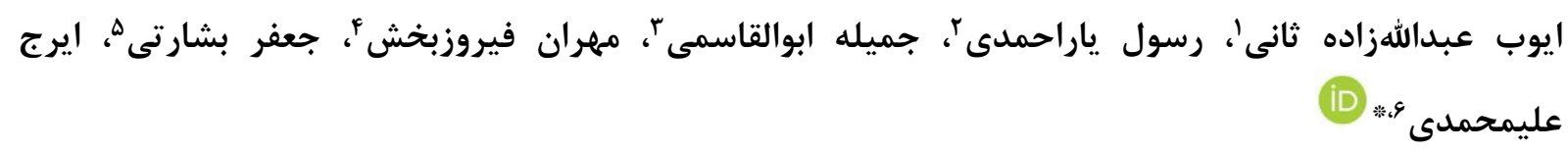
' كارشناس ارشد، گروه مهندسى بهداشت حرفهاى، دانشكده بهداشت، دانشكاه علوم يزشكى ايران، تهران، ايران

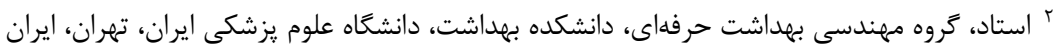

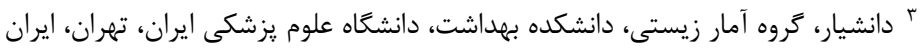

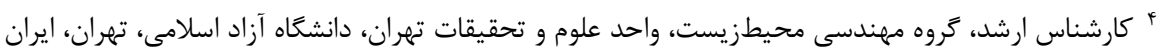

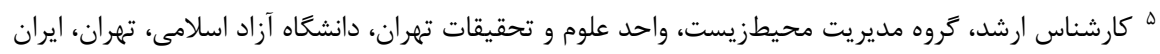

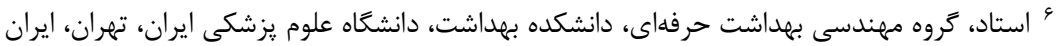

* نويسنده مسئول: ايرج عليمحمدى، گروه مهندسى بهداشت حرفهاى، دانشكده بهداشت، دانشگاه علوم يزشكى ايران، تهران، ايران. ايميل: irajrastin1@gmail.com

\section{جكيده}

سابقه و هدف: با توجه به محدوديتهاى زمانى، اجرايى و تأمين منابع مالى در هر صنعت، يكى از راهكارهاى

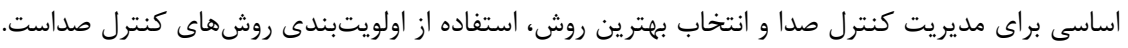

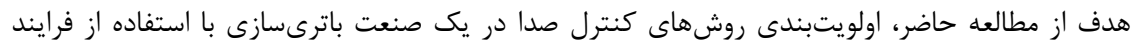
تحليل سلسله مراتبى (AHP) بود. مواد و روشها: مطالعه حاضر يك مطالعه مقطعى از نوع توصيفى-تحليلى بود. يّ از اندازهذيرى تراز فشار

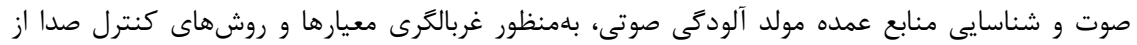

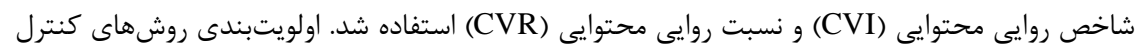

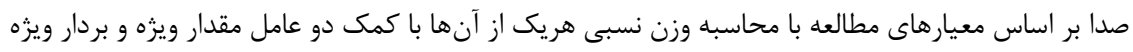

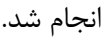
يافته ها: نتايج حاصل از مطالعه نشان داد ميزان ناساز كارى در مقايسههاى زوجى در تمام موارد كمتر از •

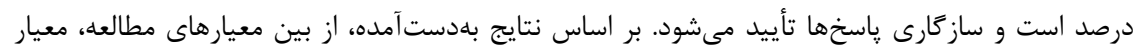

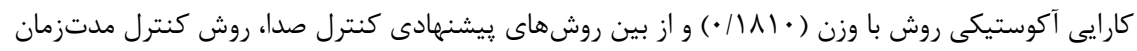

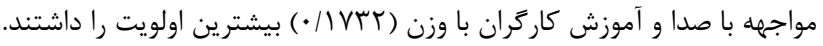

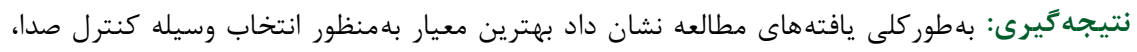

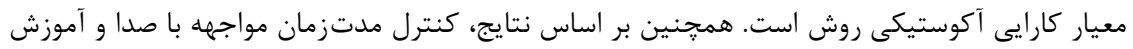

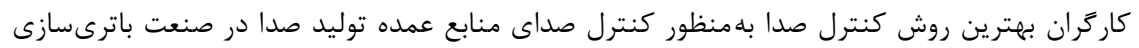

وازَّان كليدى: آلودَى صوتى؛ اولويتبندى؛ صنعت باترىسازى؛ فرايند تحليل سلسله مراتبى؛ كنترل صدا

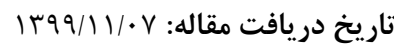
تاريخ يذيرش مقاله:

تمامى حقوق نشر براى دانشكاه علوم يزشكى همدان محفوظ است. 
صدا در يك صنعت باترىسازى است.

\section{روش كار

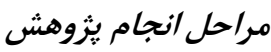

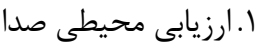

ابتدا طى بازديد و مصاحبه با مهيندسان، ناظران و كاركران

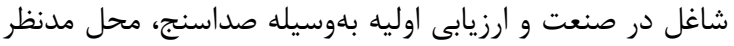

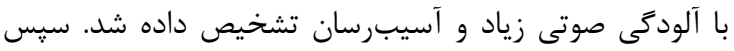

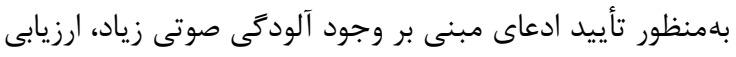
محيطى صدا مطابق استاندارد صداسنج مدل CEL -450 ساخت كشور سوئيس انجام شد. قبل صدان و بعد از اندازهخيرىها، صداسنج با استفاده از كاليبراتور كاليبره

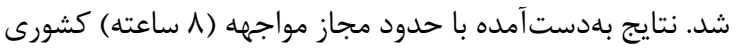
(Occupational Exposure Limit: OELs) به سه ناحيه از تراز فشار صوت (ايمن، احتياط، خطر) نقشه

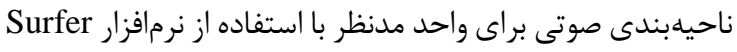

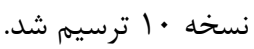

r. شناسايى و تعيين منابع عمده توليد صدا

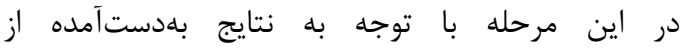

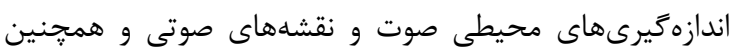
نظرخواهى از تيم خبر كان و كارشناسان خبره در مر مسائل كنترل

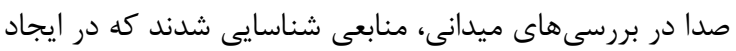

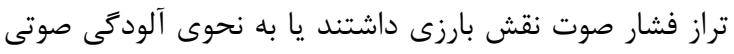
بيشترى توليد مى كردند.

r.تحليل فركانسى و تعيين تراز توان منابع توليد صداى شناسايىشده در مرحله قبل

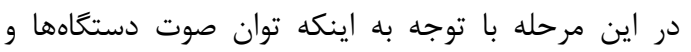

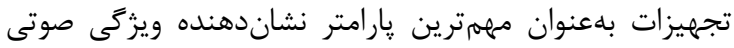

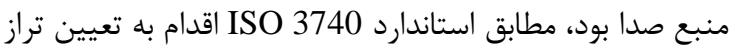

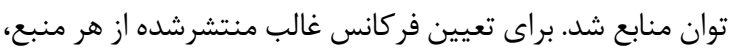
ابتدا تراز فشار صوت معادل در فر كانس هاى مركزئ برى يكى يك اكتاو باند

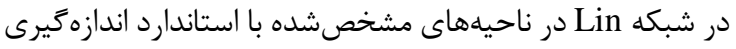

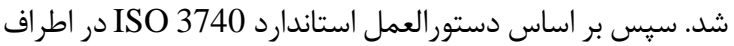
منابع شناسايىشده بر اساس ديمانسيون دستخاهها و امكان

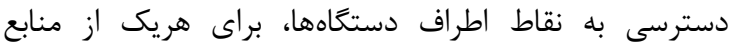
شناسايىشده تراز فشار صوت معادل تعيين و با استفاده از معادله ا تراز توان صوت براى هر منبع محاسبه شد. $\mathrm{L}_{\mathrm{w}}=\overline{\mathrm{SPL}}+10 \log \frac{\mathrm{S}}{\mathrm{S}_{0}}$

در روابط فوق: SPL ميانگين تراز فشارهاى صوت اندازه گيرىشده بر حسب dB،

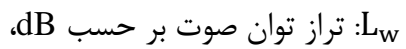

براى حفاظت از مواجهه بيشتر از حد مجاز كارگران با صدا، سه خط مشى در كنترل صدا به ترتيب اولويت بهَّونهاى است كه تا حد امكان صدا در منبع صوت كنترل شود. در اين روش

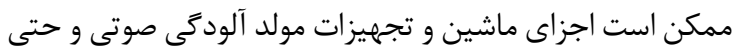

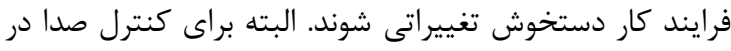

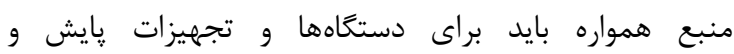
نكَهدارىهاى لازم صورت گيرد. كنترل صدا در منبع در بعضى موارد اجرايى نيست و بسيار هزينهبر است در اين مواقع كنترل

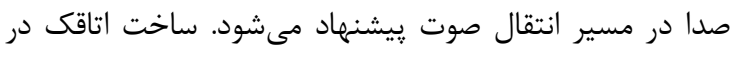

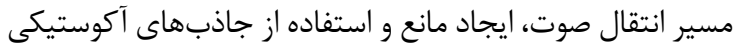

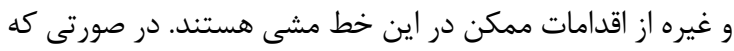

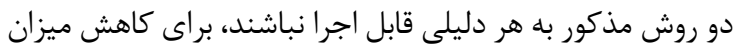

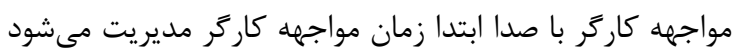

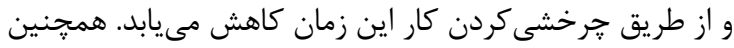

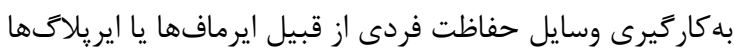

توصيه شده است [V [Y

با توجه به اينكه صنعت باترىسازى سطح وسيعى از نيروى الته

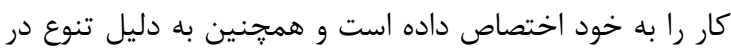

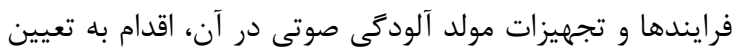

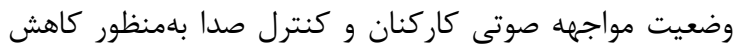

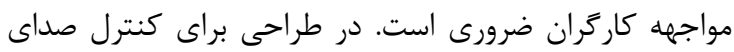
تجهيزات و اثربخشى هرجه بيشتر فرايند كاهش صدان صدا نيازمند تصميمثيرى صحيح درخصوص روش استفادهده براى كنترل

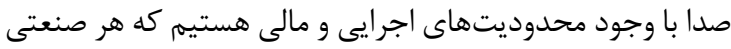

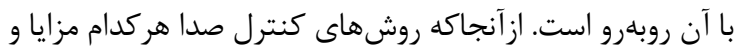

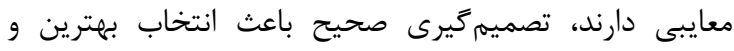

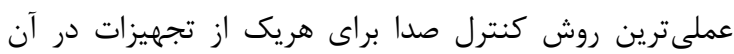

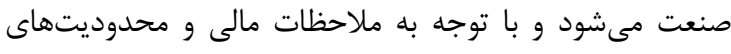

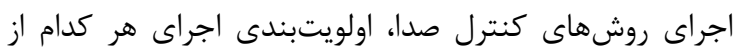

$$
\text { روشها اهميت زيادى دارد. }
$$

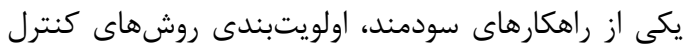

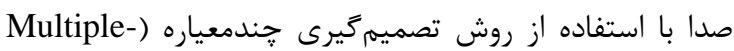
Criteria Decision Analysis: MCDM

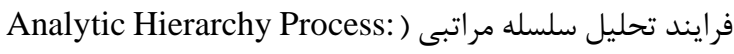

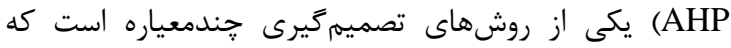

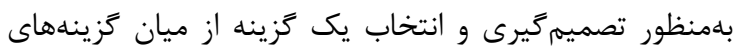

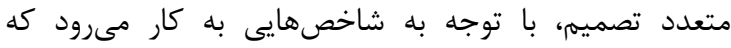

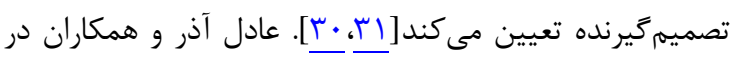
مطالعه خود، روش AHP را بهترين روش از نظر متخصصين مندان و

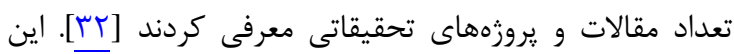

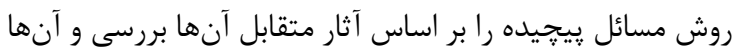

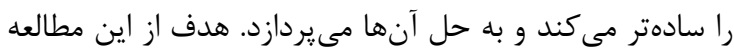
استفاده از روش AHP بلمنظور اولويتبندى روش روهاى اندي كنترل 
درختى مسئله ترسيم شد. به اين صورت كه هدف كلى مسئله

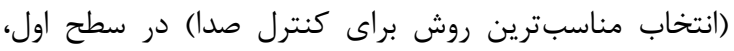

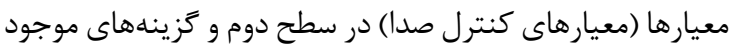
براى تصميمگيرى (روشهاى كنترل صدا) نيز در آخرين سطح

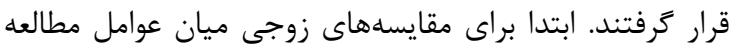

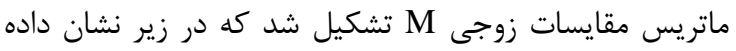

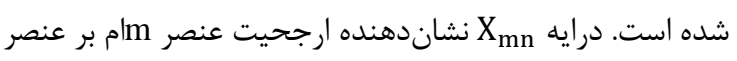
ام است كه تصميم گيرنده تعيين مى كند. (در ماتريس مقايسات

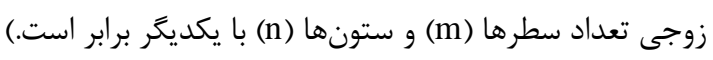

$\mathrm{M}=\left[\begin{array}{ccc}\mathrm{X}_{11} & \cdots & \mathrm{X}_{1 \mathrm{n}} \\ \vdots & \ddots & \vdots \\ \mathrm{X}_{\mathrm{m} 1} & \cdots & \mathrm{X}_{\mathrm{mn}}\end{array}\right]_{\mathrm{m} \times \mathrm{n}}$

در مرحله بعد مقادير ويزه و بردار ويزه براى ماتريس

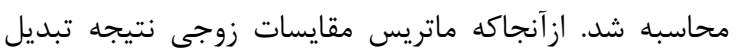
فاكتورهاى كيفى به فاكتورهاى كمى است، لذا در محاسبات حداكثر مقدار ويزه و بردار ويزه محدوده خطايى مشخص به مجاز

رابطه ميان ماتريس M و مقدار ويزه (ل) و بردار ويزه (ل) بلهورت زير است: $M \vec{v}=\lambda \vec{v}$

معادله

براى يافتن مقادير (ג) در معادله ب بايد معادله F برقرار

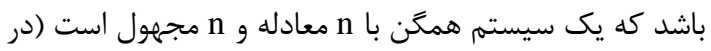
اين رابطه، (I) يك ماتريس همانى است). معادله

$M \vec{v}-\lambda \vec{v}=M \vec{v}-\lambda I \vec{v}=(M-\lambda I) \vec{v}=0$

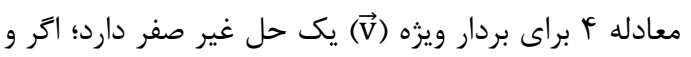
فقط اگر (معادله ه) برقرار باشد. لذا با محاسبه مقدار دترمينان بردي زير مقادير ويزه ماتريس (M) محاسبه شد.

$\operatorname{det}(\mathrm{M}-\lambda \mathrm{I})=0$

معادله ه

با محاسبه رابطه ه و بهدستآوردن مقادير ويزه ماتريس

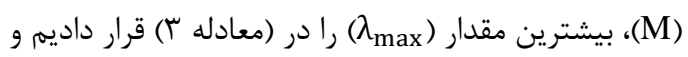

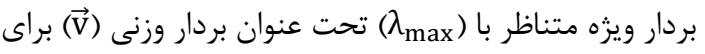
$\overrightarrow{\mathrm{v}}=\left[\begin{array}{c}\mathrm{v}_{1} \\ \vdots \\ \mathrm{v}_{\mathrm{n}}\end{array}\right]_{\mathrm{n} \times 1}$ ماتريس (M) به دست آمد. براى بهدستآوردن بردار وزنى نرمالشده، كل مقادير

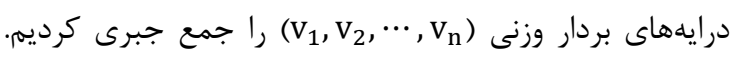
سيس مقدار هريك از درايهها بر مجموع مقادير بهدست آمده

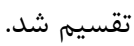

S مساحت هريك از اضلاع مكعب فرضى كه مقادير تراز فشار

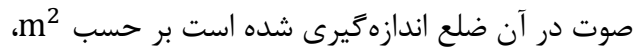

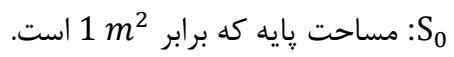

f. تهيه ليست اوليه از معيارها و روشهاى كنترل صدا بر اساس جستوجوى سيستماتيك مقالات در يايخاههاى

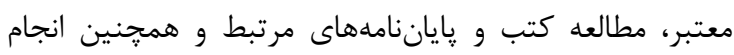
مطالعات ميدانى و با بهرهگيرى از دانش و آكاهى كارشناسان صنعت مربوطه، فهرست اوليه معيارهاى تأثير گذار بر كنترل صدا

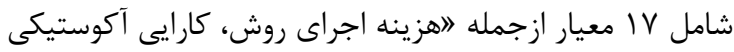
روش، قابليت اجراى روش، فضاى مورد نياز براى ساخت وسيله

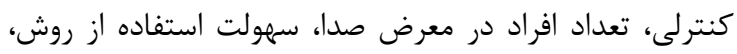

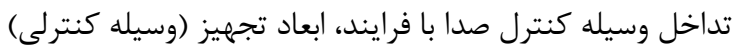

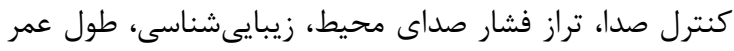

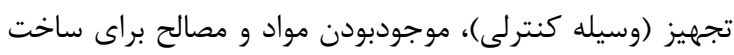

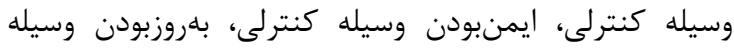

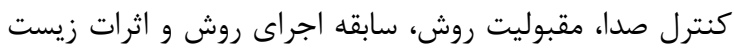

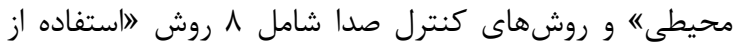
وسايل حفاظت فردى، ساخت اتاقى صوتى روى منابع توليد صدا،

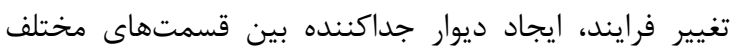

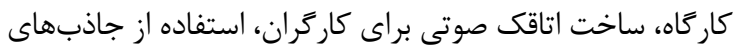

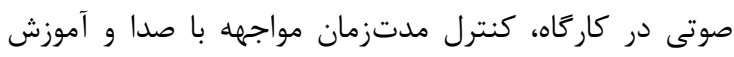

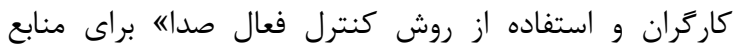

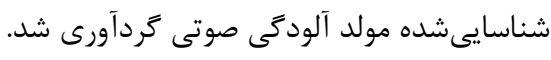

ه. انتخاب معيارهاى كليدى در كنترل صدا

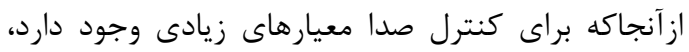

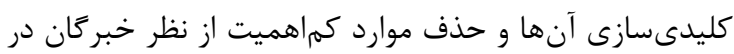

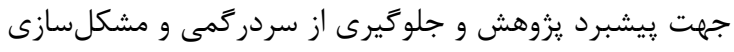

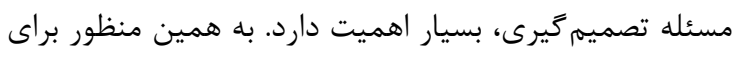
تعيين معيارهاى كليدى دركنترل صدا، فهرست اوليه معيارها

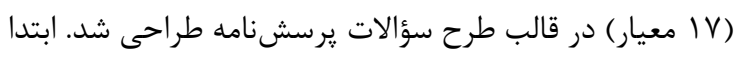

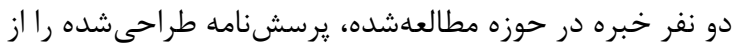

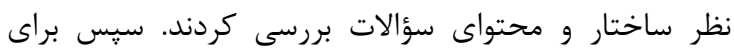

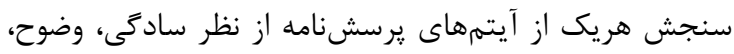

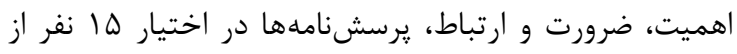
تيم خبر گان قرار گرفت. سيس شاخص روايى محتو ايى (CVI)

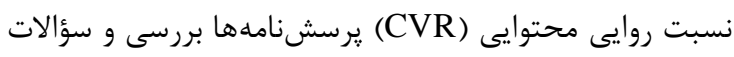

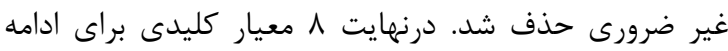

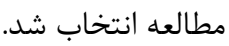

צ. اجراى فرايند تحليل سلسله مراتبى (AHP)

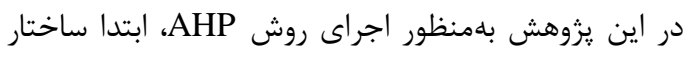


جدول ا: شاخص ساز كارى تصادفى استفادهده در روش AHP بر اساس تعداد خبركان

\begin{tabular}{|c|c|c|c|c|c|c|c|c|c|c|c|c|c|c|c|}
\hline 10 & If & rו & r & 11 & 1. & 9 & $\Lambda$ & $v$ & 9 & $\Delta$ & $p$ & $r$ & $r$ & 1 & $\mathbf{N}$ \\
\hline $1 / \Delta \Lambda$ & $1 / \Delta V$ & $1 / \Delta \Delta$ & س/D & $1 / \Delta 1$ & $1 / F \wedge$ & $1 / F F$ & $1 / 4$ & $1 / \mu F$ & $1 / T F$ & $1 / 1$. & $\cdot \mid \Lambda \Lambda$ & . $/ \Delta T$ & . & . & RI \\
\hline
\end{tabular}

آلودگى صوتى قرار داشتند و تراز فشار صوت در هيج نقطهاى از

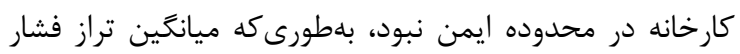

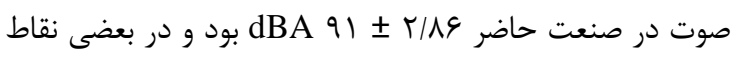

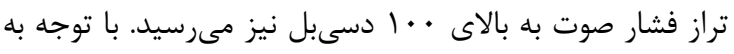

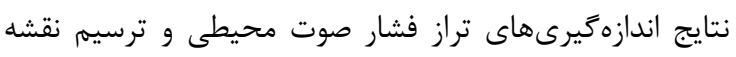

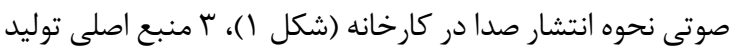

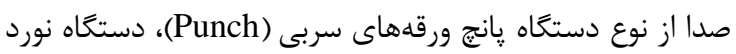
ورقههاى سربى (Rolling) و دستخاه اكسيند يانج ورقههاى

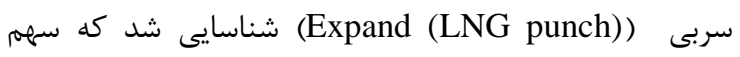
عمدهاى در توليد آلودكى صوتى داشتند.

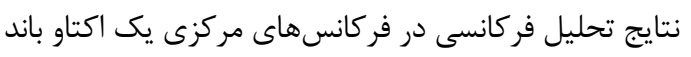

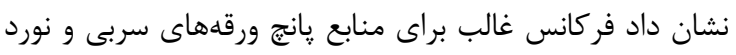

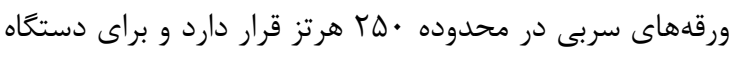

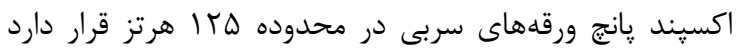

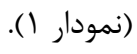

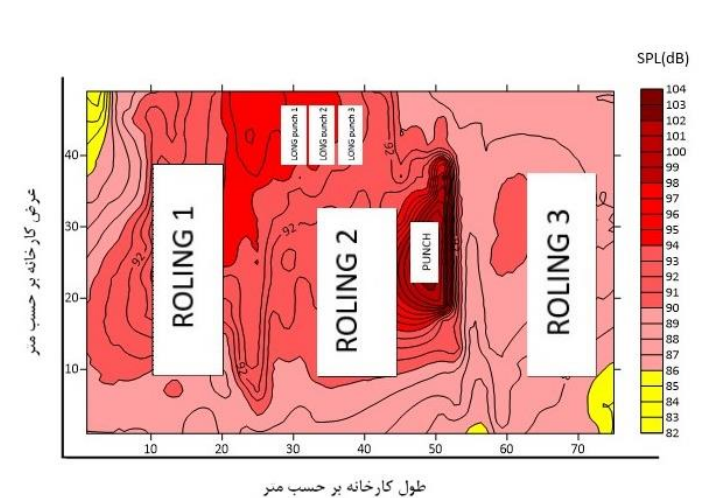

شكل ا: نقشه صوتى حاصل از نقاط اندازهيرىشده با نرمافزار نسخه · ا و محل قراركيرى دستخاهها در كارخانه $\vec{v}($ normalized $)=\left[\begin{array}{c}\frac{v_{1}}{v_{1}+v_{2}+\cdots+v_{n}} \\ \vdots \\ \frac{v_{n}}{v_{1}+v_{2}+\cdots+v_{n}}\end{array}\right]_{n \times 1}$

در مرحله بعد نرخ سازگارى (Consistency Ratio: CR)

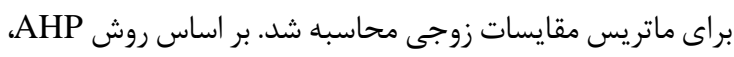

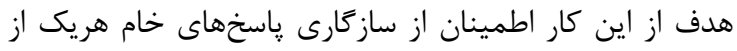

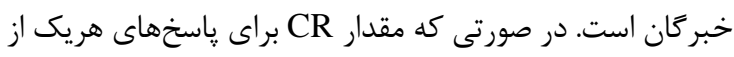

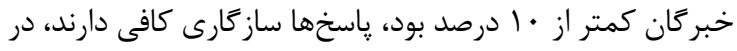

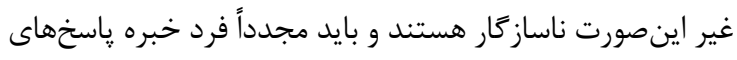

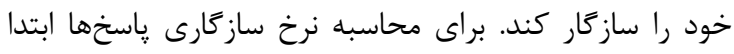

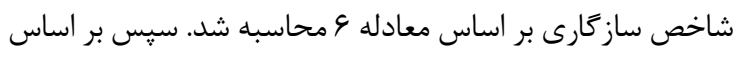

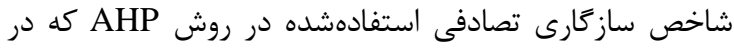

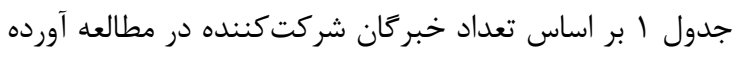

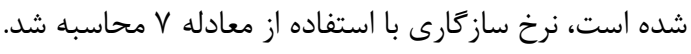
$\begin{array}{ll}\mathrm{CI}=\frac{\lambda_{\max }-\mathrm{n}}{\mathrm{n}-1} & \text { S مe }\end{array}$ جدول شاخص ساز₹ارى تصادفى بر اساس تعداد خبركان شركت كننده در مطالعه حاضر در زير آمده است [سباري]. نتايج

\section{نتايج ارزيابسى محيطى صدا}

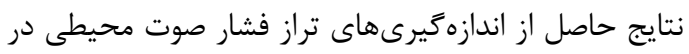

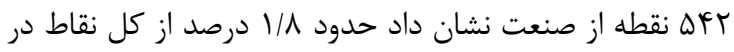

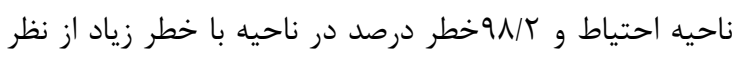

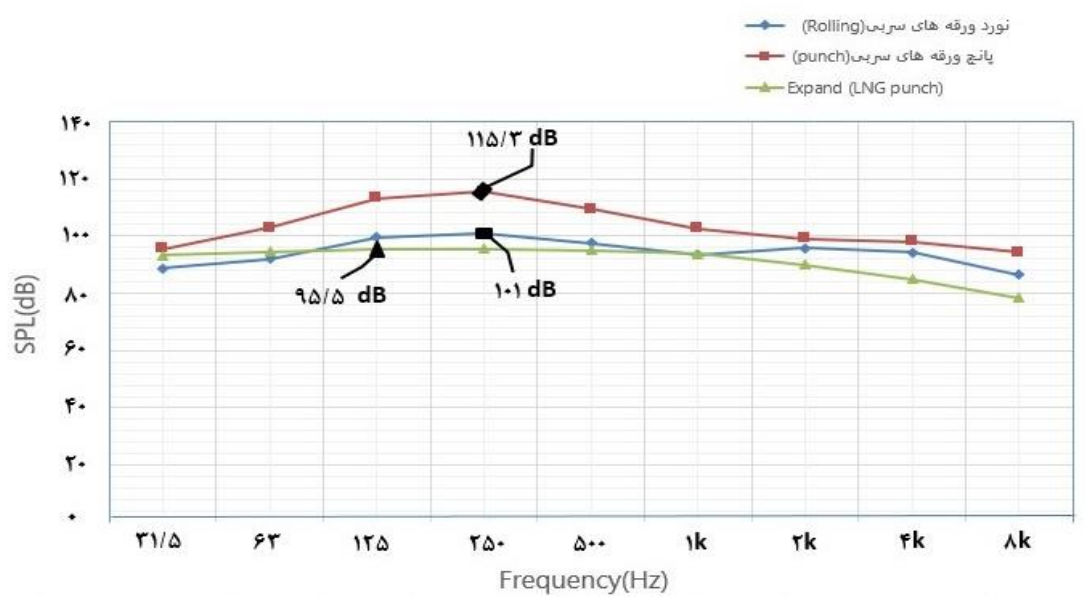

نمودار ا: نتايج تحليل فركانسى در فركانسهاى مركزى يك اكتاو باند 
و روشهاى كنترلى، نرخ سازگارى براى هريك از يرسشنامهها

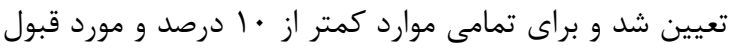

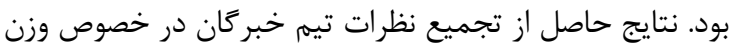

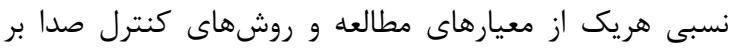

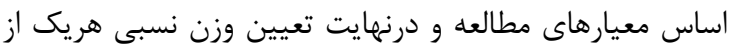

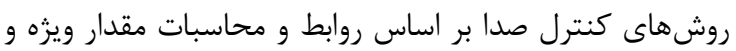
بردار ويزه در جداول زير آمده است.

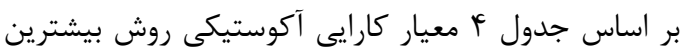

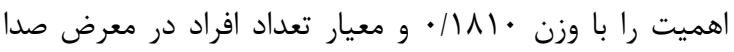
كمترين اهميت را با وزن 9 •1 • • • دارد

\section{نتا يج حاصل /ز تعيين معيارهاى كليدى و روشهاى كنترل}

صدا

يس از بررسى نسبت روايى محتوايى و شاخص روايى محتوايى IV معيار كنترل صدا، بر اساس جدول لاوشه 1 م معيار

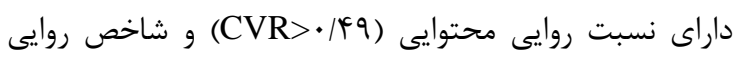

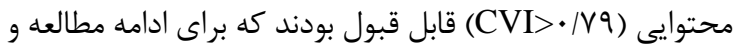
بررسى در فرايند تحليل سلسله مراتبى (AHP) باقى ماندند.

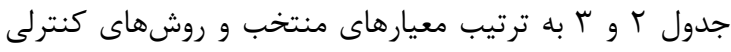
ييشنهادى هستند. يس از اظهارنظر تيم خبركان در خصوص هريك از معيارها

\begin{tabular}{|c|c|c|}
\hline كد مربوطه & معيارهاى كنترل صدا & رديف \\
\hline CRA & هزينه اجراى روش & 1 \\
\hline CRB & كار ايى آكوستيكى روش & r \\
\hline CRC & قابليت اجراى روش & r \\
\hline CRD & تعداد افراد در معرض صدا & f \\
\hline CRE & تراز فشار صداى محيط & $\Delta$ \\
\hline $\mathrm{CRF}$ & موجودبودن مواد و مصالح براى ساخت وسيله كنترلى & \& \\
\hline CRG & ايمنبودن وسيله كنترلى & v \\
\hline $\mathrm{CRH}$ & تداخل وسيله كنترل صدا با فرايند & $\wedge$ \\
\hline
\end{tabular}

\begin{tabular}{|c|c|c|}
\hline كد مربوطه & روشهاى كنترل صدا & رديف \\
\hline NCMA & استفاده از وسايل حفاظت فردى & 1 \\
\hline NCMB & ساخت اتاقى صوتى روى منابع توليد صدا & r \\
\hline NCMC & تغيير فرايند & r \\
\hline NCMD & ايجاد ديوار جداكننده بين قسمتهاى مختلف كاركاه & r \\
\hline NCME & ساخت اتاقى صوتى براى كاركران & $\Delta$ \\
\hline $\mathrm{NCMF}$ & استفاده از جاذبهاى صوتى در كارگًاه & 4 \\
\hline NCMG & كنترل مدتزمان مواجهه با صدا و آموزش كاركران & $\checkmark$ \\
\hline $\mathrm{NCMH}$ & استفاده از روش كنترل فعال صدا & $\wedge$ \\
\hline
\end{tabular}

جدول \&ٔ: وزن معيارهاى مطالعه بر اساس تجميع نظرات خبركان

\begin{tabular}{|c|c|c|c|c|c|c|c|c|c|c|}
\hline \multirow[b]{2}{*}{ معيارها } & \multirow[b]{2}{*}{ CRA } & \multirow[b]{2}{*}{ CRB } & \multirow[b]{2}{*}{ CRC } & \multirow[b]{2}{*}{ CRD } & \multirow[b]{2}{*}{ CRE } & \multirow[b]{2}{*}{ CRF } & \multirow[b]{2}{*}{ CRG } & \multirow[b]{2}{*}{ CRH } & \multicolumn{2}{|c|}{$\lambda_{\max }=\wedge / \cdot 41$} \\
\hline & & & & & & & & & بردارهاى ويزه & وزن نهايى \\
\hline CRA & 1 & .1994 & $\cdot / \Lambda \Delta$. & $1 / V \Lambda$. & 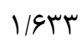 & $1 / 7 / 9$ & $1 / r r q$ & $1 / 1 \cdot 0$ & $\cdot / \mu \Lambda$. &. $\mid 1 \mathrm{HA}$ \\
\hline CRB & $1 / \Delta \cdot V$ & 1 & I/TTY & $T /|9|$ & $r / \cdot r_{\Lambda}$ & $1 / T F V$ & $1 / 499$ & I/DHV &.$/ 491$ &.$|11|$. \\
\hline $\mathrm{CRC}$ & I/IVV & $\cdot|\lambda| \Lambda$ & 1 & l/gF. & l/GFT & $1 / T \Delta \Lambda$ & $1 / r \cdot r$ & $1 / 4 v q$ & $\cdot / F T$. & - IIOTV \\
\hline CRD & - IDGT & . &.$|9|$. & 1 & . & .1099 &.$/ 190$ & .1991 & - ITTY & $\cdot 1 \cdot 1 \cdot 9$ \\
\hline CRE & .1911 & $\cdot / 4 \wedge \Lambda$ &.$|\Delta| \Delta$ & I/DVG & 1 & $.19 \cdot 9$ & $\cdot \mid \mathrm{ANQ}$ & $\cdot 10 \% \Delta$ & $\cdot / r \Delta \Delta$ & $.1 .9 \mathrm{TV}$ \\
\hline CRF & - $|A T|$ & - MFT &.$/ 19 \Delta$ & $1 / 999$ & $1 / 1 \ldots$ & 1 & $. / 91)^{f}$ & $\cdot / V \Delta S$ & $\cdot|r| f$ & $.|\|| f \mid$ \\
\hline $\mathrm{CRG}$ & - IVAT & $.199 \mathrm{~V}$ & . & $1 / 11 \mathrm{~V}$ & $1 / 1 r q$ & $1 / .94$ & 1 & $1 / . .9$ & . & TIIIT \\
\hline $\mathrm{CRH}$ & $\cdot 19 \cdot \Delta$ & $\cdot|90|$ & - IVTD & I/FFV & $1 / V V I$ & I/TTY &.$/ 994$ & 1 & $\cdot / \pi \Delta \cdot$ &.$/ 1 T V T$ \\
\hline & & & & & & & & & $A v g=r / v<q$ & - \\
\hline
\end{tabular}


جدول ه: وزن هريك از روشهاى كنترل صدا بر اساس تجميع نظرات خبركان

\begin{tabular}{|c|c|c|c|c|c|c|c|c|c|c|}
\hline \multirow[b]{2}{*}{ ت تداخل } & \multirow[b]{2}{*}{ NCMA } & \multirow[b]{2}{*}{ NCMB } & \multirow[b]{2}{*}{ NCMC } & \multirow[b]{2}{*}{ NCMD } & \multirow[b]{2}{*}{ NCME } & \multirow[b]{2}{*}{ NCMF } & \multirow[b]{2}{*}{ NCMG } & \multirow[b]{2}{*}{ NCMH } & \multicolumn{2}{|c|}{$\lambda_{\max }=\Lambda / 19 \mathrm{~F}$} \\
\hline & & & & & & & & & بردارهاى ويزه & وزن نهايى \\
\hline NCMA & 1 & $1 / .9 T$ & I/TAY & $1 / \cdot \varphi_{1} \Lambda$ & $1 / T \Delta q$ & $1 / 11 \pi$ & $.19 V T$ & $1 / 94 \wedge$ & $\cdot \pi v \Delta$ & R \\
\hline NCMB &.$|94|$ & 1 & $1 / 90$. & .1994 & 1/11 & .1910 & $\cdot / V F V$ & T/ITT & $\cdot|r V|$ & $. / 1 r<q$ \\
\hline NCMC & $\cdot / \mathrm{r}$. & .19 .9 & 1 & . IGTr & $\cdot 1 \Delta \Delta 9$ & - IGTA &.$|8| r$ & $1 / 4 \wedge 1$ & $\cdot / T F \wedge$ &.$/ \cdot 9 \cdot r$ \\
\hline NCMD &.$/ 9 \Delta \Delta$ & $1 / \cdots 4$ & $1 / 9 \cdot 9$ & 1 & $1 / 1 T$. & . IAFT & $\cdot 19 \wedge \Delta$ & $r / \cdot r \Delta$ & אצr & AMIM \\
\hline NCME & - /vqF & $\cdot 1199$ & 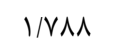 & ./^9r & 1 & $\cdot 11 \cdot 9$ & $\cdot / V 11$ & $r / \cdot \Delta V$ & $\cdot / \mu F \Delta$ & $\cdot / 1 r \Delta S$ \\
\hline NCMF & $\cdot 1199$ & 1/.9r & 1/ब१ & $1 / 119$ & $|/ T F|$ & 1 & سMr & $1 / 911$ & $\cdot /$ rAF & . /1199 \\
\hline NCMG & I/FAV & גז"ו & 1/צra & $1 / 49$. & $1 / 4 \cdot V$ & $1 /$ KGF & 1 & $1 / 9 \cdot 1$ & . IFVG & ./IVTr \\
\hline NCMH & س & $\cdot / 4 \& q$ & $\cdot 19 \vee \Delta$ &.$/ 4 q 4$ & $\cdot / 4 \wedge s$ & $\cdot|\Delta T|$ & . DOTE & 1 & .1119 &.$/ .9 \wedge \mathrm{V}$ \\
\hline & & & & & & & & & $A v g=r / V \Delta$. & - \\
\hline
\end{tabular}

فهمتر نسبت به مفاهيم كيفى [عب]، براى حل مشكلات ناشى از

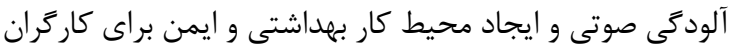

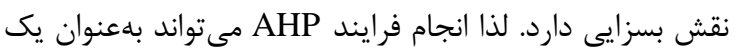

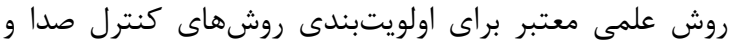

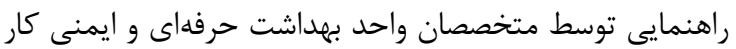

$$
\text { مورد استفاده قرار كيرد. }
$$

در اين مطالعه بر اساس نتايج حاصل از مقايسه معيارها از

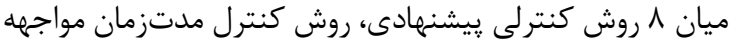

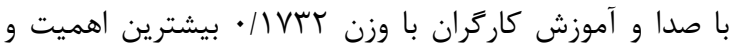
روش كنترل فعال صدا با وزن \&AV•

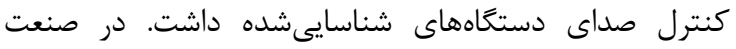

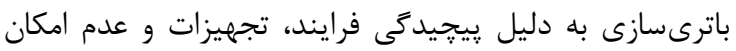

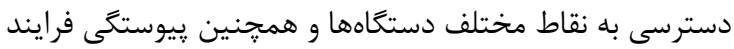
و در اغلب موارد مجزانبودن تكتك دستكاهنها، امكان اعمال كنترلهاى مهندسى دشوار است. لذا يكى از از دلايلى كه

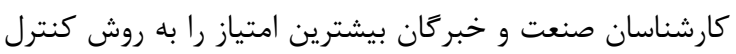

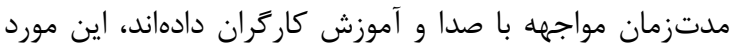

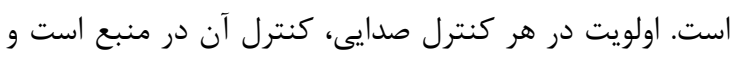

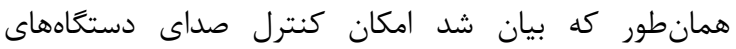

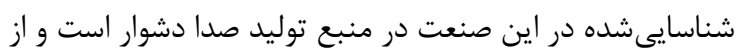

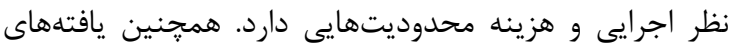

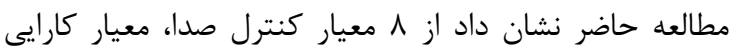

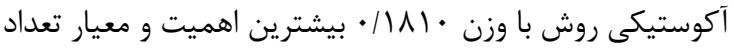

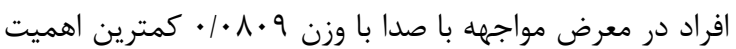

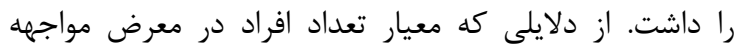

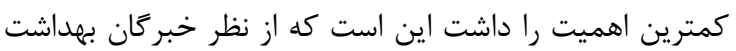

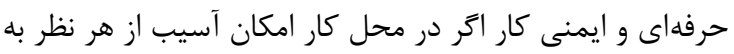

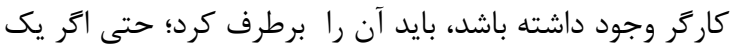

$$
\text { كاركر در محل كار وجود داشته باشد. }
$$

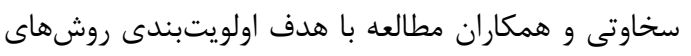

كنترل و كاهش آلودكى صدا در شركت سيمان لارستان با هدفان
مطابق جدول ه و بر اساس تجميع نظرات خبركان، روش

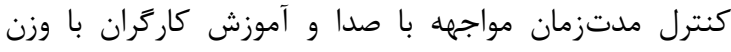

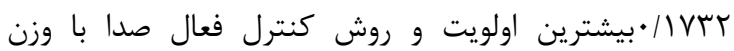
4NV آلودכى صوتى در صنعت مطالعهشده دارند.

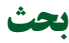

مطالعه حاضر با هدف اولويتبندى روشهاى كنترل صدا در در بردي يكى صنعت باترىسازى با استفاده از فرايند تحليل سلسله مراتبى راتي

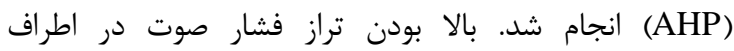

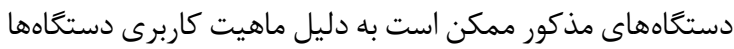

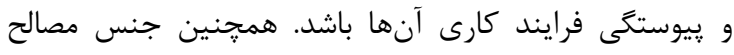

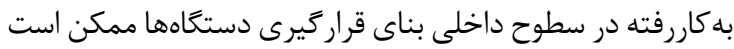

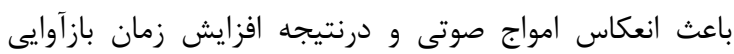

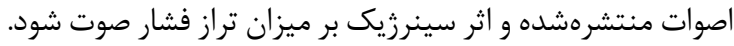

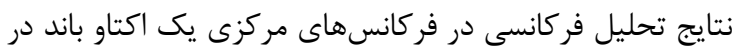

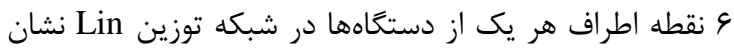

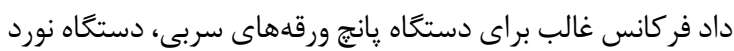

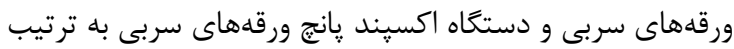

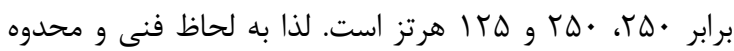

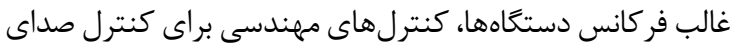

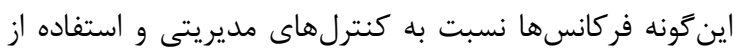
تجهيزات حفاظت فردى ارجحيت و كارايى بيشترى دارد

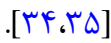
فرايند تحليل سلسله مراتبى (AHP) بهعنوان روشى كارآمد با فرمولهكردن مسئله بهصورت سلسله مراتبى، درنظركرفتئن

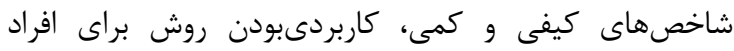
متخصص، ادغام نظرات متفاوت و تعيين كزينه كاينه نهايى، استفاديه

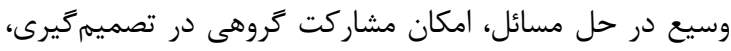

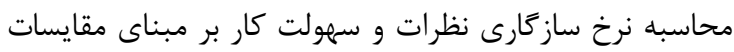

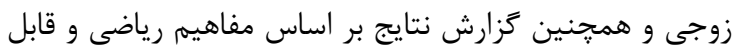


همجنين بر اساس نتايج، كنترل مدتزمان مواجهه با صدا و

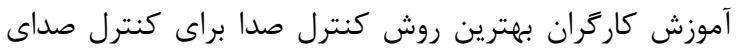
منابع عمده توليد صدا در صنعت باترىسازى بودن رونت

\section{تشكر و قلرو مانى}

مطالعه حاضر حاصل بخشى از طرح يزوهشى با كد اخلاق

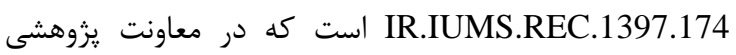

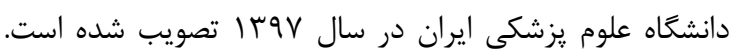

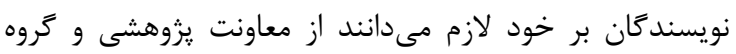

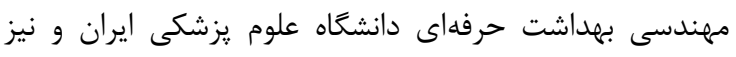

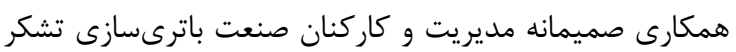

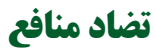

در اين يُزوهش، هيجگَونه تضاد منافع و تعارضى توسط

$$
\text { نويسندكان وجود ندارد. }
$$

\section{ملاحظات اخلاقى}

در اين :زووهش، كليه ملاحظات اخلاقى مورد تاييد دانشعاه

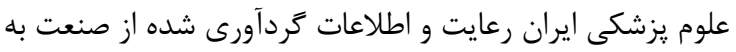
صورت محرمانه حفظ شده است. كد اخلاق اين مطالعه IR.IUMS.REC.1397.174 محتدانه حفظ است.

\section{سمم نويسندكان}

ايوب عبدالله زاده ثانى: كردآورى پايان نامه و تهيه كننده اصلى

مقاله رسول ياراحمدى: استاد مشاور در تهيه ياياننامه جميله ابوالقاسمى: استاد مشاور در تهيه يايان نامده

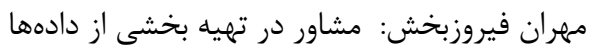
جعفر بشارتى: مشاور در تهيه بخشى از دادهها

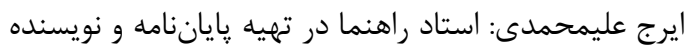

تمام حمايتهاى مالى اين طرح كه بخشى از پاياننامه

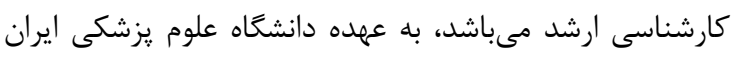

\section{REFERENCES}

1. Atmaca E, Peker I, Altin A. Industrial noise and its effects on humans. Polish J Environ Stud. 2005;14(6):721-6.

2. van Kempen E, van Kamp I, Fischer P, Davies H, Houthuijs D, Stellato R, et al. Noise exposure and children's blood pressure and heart rate: the RANCH project. Occup Environ Med. 2006;63(9):632-9. PMID: 16728500 DOI: 10.1136/oem.2006.026831
استفاده از روش AHP يزوهشى انجام دادند. نتايج مطالعه آنها نشان داد مناسبترين روش كنترل در صنعت مطالعهشده،

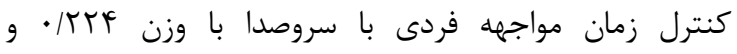

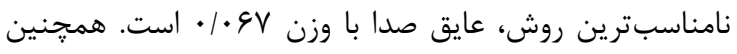

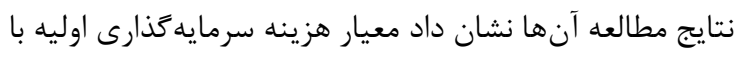

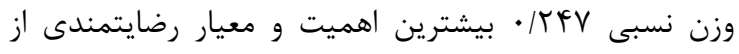

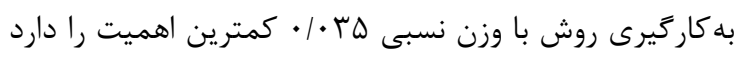

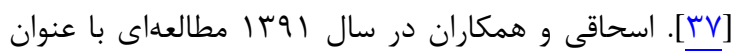

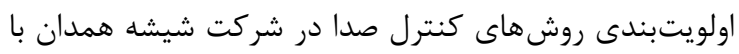

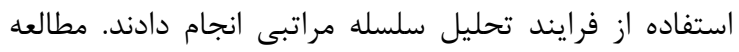

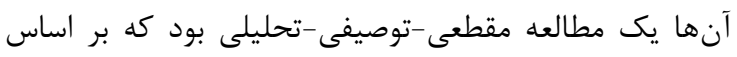

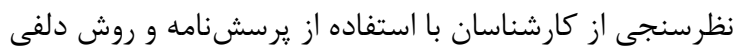

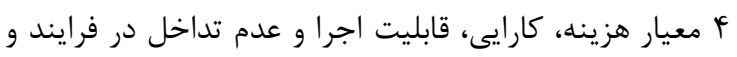

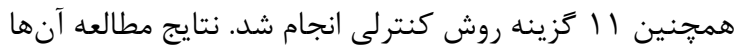

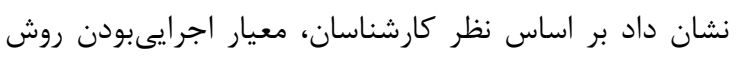

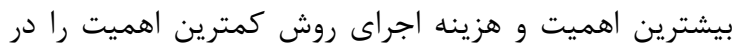

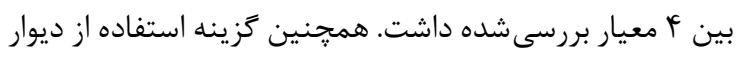
جداكننده كامل بين دو بخش اصلى به همراه استفاده از مواد

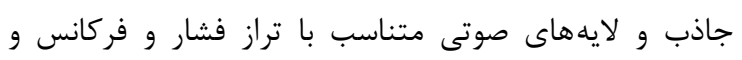

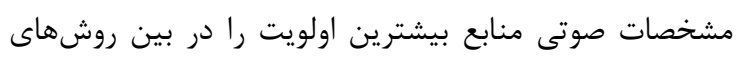
بررسى شده داشت [1ی]. در هر دو مطالعه مذكور براى غربالكرى معيارها و روشها دها به دانه

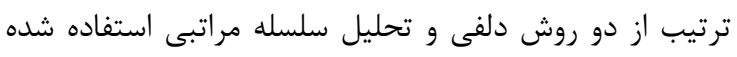

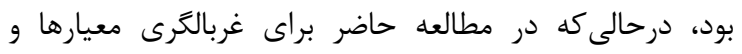

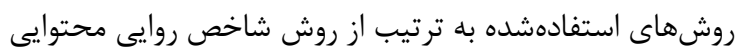

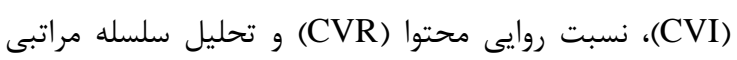

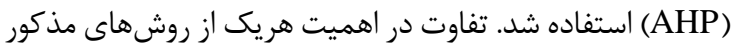

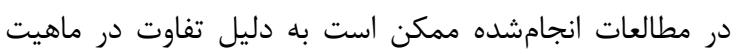

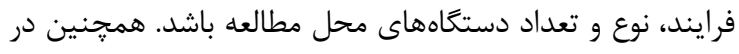

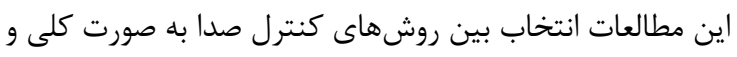

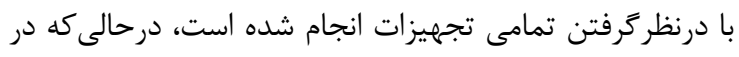

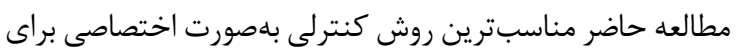

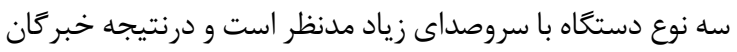
در ارائه نظرات خود متمركزتر و اختصاصى دتر عمل مى بـ كنند.

\section{نتيجه كيرى}

بلهطوركلى يافته هاى مطالعه نشان داد بهترين معيار براى

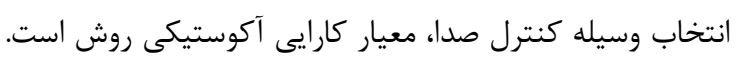

3. Kraus U, Schneider A, Breitner S, Hampel R, Rückerl R, Pitz $\mathrm{M}$, et al. Individual daytime noise exposure during routine activities and heart rate variability in adults: a repeated measures study. Environ Health Perspect. 2013;121(5):60712. PMID: 23512292 DOI: $10.1289 /$ ehp. 1205606

4. Burns KN, Sun K, Fobil JN, Neitzel RL. Heart rate, stress, and occupational noise exposure among electronic waste recycling 
workers. Int J Environ Res Public Health. 2016;13(1):140. PMID: 26797626 DOI: 10.3390/ijerph13010140

5. Münzel T, Sørensen M, Schmidt F, Schmidt E, Steven S, Kröller-Schön S, et al. The adverse effects of environmental noise exposure on oxidative stress and cardiovascular risk. Antioxid Redox Signal. 2018;28(9):873-908. PMID: 29350061 DOI: $10.1089 /$ ars. 2017.7118

6. Samson J, Sheeladevi R, Ravindran R. Oxidative stress in brain and antioxidant activity of Ocimum sanctum in noise exposure. Neurotoxicology. 2007;28(3):679-85. PMID: 17379314 DOI: $10.1016 /$ j.neuro.2007.02.011

7. Åhrlin U. Activity disturbances caused by different environmental noises. J Sound Vib. 1988;127(3):599-603. DOI: 10.1016/0022-460X(88)90389-6

8. Phoon WH, Lee HS, Chia SE. Tinnitus in noise-exposed workers. Occup Med. 1993;43(1):35-8. PMID: 8422445 DOI: $10.1093 /$ occmed/43.1.35

9. Janssen SA, Vos H, Eisses AR, Pedersen E. A comparison between exposure-response relationships for wind turbine annoyance and annoyance due to other noise sources. $J$ Acoust Soc Am. 2011;130(6):3746-53. PMID: 22225031 DOI: $10.1121 / 1.3653984$

10. Eze IC, Foraster M, Schaffner E, Vienneau D, Héritier H, Pieren R, et al. Transportation noise exposure, noise annoyance and respiratory health in adults: a repeatedmeasures study. Environ Int. 2018;121(Pt 1):741-50. PMID: 30321849 DOI: 10.1016/j.envint.2018.10.006

11. Leung TM, Chau CK, Tang SK, Xu JM. Developing a multivariate model for predicting the noise annoyance responses due to combined water sound and road traffic noise exposure. Appl Acoust. 2017;127:284-91. DOI: 10.1016/j.apacoust.2017.06.020

12. Alimohammadi I, Sandrock S, Gohari MR. The effects of low frequency noise on mental performance and annoyance. Environ Monit Assess. 2013;185(8):7043-51. PMID: 23338951 DOI: $10.1007 / \mathrm{s} 10661-013-3084-8$

13. Sygna K, Aasvang GM, Aamodt G, Oftedal B, Krog NH. Road traffic noise, sleep and mental health. Environ Res. 2014;131:17-24. PMID: 24637180 DOI: 10.1016/j.envres. 2014.02.010

14. Halperin D. Environmental noise and sleep disturbances: a threat to health? Sleep Sci. 2014;7(4):209-12. PMID: 26483931 DOI: $10.1016 /$ j.slsci.2014.11.003

15. Zaharna M, Guilleminault C. Sleep, noise and health. Noise Health. 2010;12(47):64. DOI: 10.4103/1463-1741.63205

16. Héritier H, Vienneau D, Foraster M, Eze IC, Schaffner E, Thiesse L, et al. Diurnal variability of transportation noise exposure and cardiovascular mortality: a nationwide cohort study from Switzerland. Int $J$ Hyg Environ Health. 2018;221(3):556-63. PMID: 29482991 DOI: 10.1016/j.ijheh. 2018.02 .005

17. Münzel T, Gori T, Babisch W, Basner M. Cardiovascular effects of environmental noise exposure. Eur Heart J. 2014;35(13):82936. PMID: 24616334 DOI: 10.1093/eurheartj/ehu030

18. Tomei G, Fioravanti M, Cerratti D, Sancini A, Tomao E, Rosati M V, et al. Occupational exposure to noise and the cardiovascular system: a meta-analysis. Sci Total Environ. 2010;408(4):681-9. PMID: 19931119 DOI: 10.1016/j. scitotenv.2009.10.071

19. Ristovska G, Laszlo HE, Hansell AL. Reproductive outcomes associated with noise exposure-a systematic review of the literature. Int J Environ Res Public Health. 2014;11(8):793152. PMID: 25101773 DOI: 10.3390/ijerph110807931

20. Barber JR, Crooks KR, Fristrup KM. The costs of chronic noise exposure for terrestrial organisms. Trends Ecol Evol. 2010;25(3):180-9. PMID: 19762112 DOI: 10.1016/j.tree. 2009.08.002

21. Picard M, Girard SA, Simard M, Larocque R, Leroux T, Turcotte F. Association of work-related accidents with noise exposure in the workplace and noise-induced hearing loss based on the experience of some 240,000 person-years of observation. Accid Anal Prev. 2008;40(5):1644-52. PMID: 18760091 DOI: 10.1016/j.aap.2008.05.013

22. Girard SA, Picard M, Davis AC, Simard M, Larocque R, Leroux T, et al. Multiple work-related accidents: tracing the role of hearing status and noise exposure. Occup Environ Med. 2009;66(5):319-24. PMID: 19174422 DOI: 10.1136/ oem.2007.037713

23. Cantley LF, Galusha D, Cullen MR, Dixon-Ernst C, Rabinowitz PM, Neitzel RL. Association between ambient noise exposure, hearing acuity, and risk of acute occupational injury. Scand J Work Environ Health. 2015;41(1):75-83. PMID: 251375565 DOI: 10.5271/sjweh.3450

24. Taqizade S, Eskandari H, Alimohammadi I, Jaderi F. A fuzzy expert system for selection of an effective method for noise reduction in a petrochemical complex. Noise Control Eng J. 2014;62(5):344-53. DOI: 10.3397/1/376233

25. Barron RF. Industrial noise control and acoustics. Florida: CRC Press; 2002.

26. Hansen CH. Fundamentals of acoustics. Occupational exposure to noise: evaluation, prevention and control. Geneva: World Health Organization; 2001. P. 23-52.

27. Goelzer B, Hansen CH, Sehrndt G. Occupational exposure to noise: evaluation, prevention and control. Geneva: World Health Organisation; 2001

28. Hobbs BF, Meier PM. Multicriteria methods for resource planning: an experimental comparison. IEEE Trans Power Syst. 1994;9(4):1811-7. DOI: 10.1109/59.331435

29. Triantaphyllou E. Multi-criteria decision making methods. Multi-criteria decision making methods: a comparative study. Boston, MA: Springer; 2000. P. 5-21.

30. Saaty TL. Decision making with the analytic hierarchy process. Int J Serv Sci. 2008;1(1):83-98. DOI: 10.1504/ IJSSci.2008.01759

31. Pal S, Bhadane MS, Babu C. Analytical hierarchical process: fundamentals and its application. New York: McGraw-Hill; 2015.

32. Azar A, Vafaei F. Ranking MADM multi-index decision making techniques using some multi-criteria decision making methods in fuzzy environment and comparing it with DEA ranking. Manag Achiev. 2010;7(41):21-38.

33. Alonso JA, Lamata MT. Consistency in the analytic hierarchy process: a new approach. Int J Uncertainty Fuzzin Knowl Based Syst. 2006;14(4):445-59.

34. Baliatsas C, van Kamp I, van Poll R, Yzermans J. Health effects from low-frequency noise and infrasound in the general population: Is it time to listen? A systematic review of observational studies. Sci Total Environ. 2016;557:163-9. DOI: 10.1142/S0218488506004114

35. Bolin K, Bluhm G, Eriksson G, Nilsson ME. Infrasound and low frequency noise from wind turbines: exposure and health effects. Environ Res Lett. 2011;6(3):35103. DOI: 10.1088/1748-9326/6/3/035103

36. Atthirawong W, MacCarthy B. An application of the analytical hierarchy process to international location decision-making. The 7th Annual Cambridge International Manufacturing Symposium: Restructuring Global Manufacturing, University of Cambridge, Cambridge, England; 2002. P. 1-18.

37. Sekhavati E, Mohammadi ZM, Mohammad FI, Faghihi ZA. Prioritizing methods of control and reduce noise pollution in Larestan cement Factory using analytical hierarchy process (AHP). Toloo-E-Behdasht. 2014;13(2):156-67.

38. Mahboobe E, Golmohamadi R, Riahi-Korram M. Prioritizing of noise control methods in the Hamadan glass company by the analytical hierarchy process (AHP). J Occup Health Saf. 2012;2(1):75-84 